\title{
AÇÕES TERRITORIAIS EM CENAS DE USO DE DROGAS NO RIO DE JANEIRO: UM CENÁRIO DE MUDANÇAS NA PANDEMIA DA COVID-19
}

DRUG CONSUMPTION ENVIRONMENTS IN RIO DE JANEIRO: A CHANGING SCENARIO OVER COVID-19 PANDEMICS

\section{Beatriz Brandão}

Pesquisadora de pós-doutorado em Sociologia da Cultura pela Universidade de São Paulo (USP). Doutora em Ciências Sociais pela Pontifícia Universidade Católica do Rio de Janeiro (PUC-RIO). Mestra em Ciências Sociais pela Universidade do Estado do Rio de Janeiro (UERJ). Pesquisadora do Instituto de Pesquisa Econômica Aplicada (IPEA) na pesquisa sobre metodologias de cuidado a usuários problemáticos de drogas (2019-2020).

\section{Rogério da Silva Ferreira}

Enfermeiro, especialista em Enfermagem Psiquiátrica e Saúde Mental pela Universidade do Estado do Rio de Janeiro (UERJ). Diretor do Centro de Atenção Psicossocial Álcool e Outras Drogas (CAPS AD III) Antônio Carlos Mussum e da Unidade de Acolhimento de Adultos (UAA Cacildis). Vice-presidente do capítulo brasileiro International Nurses Society on Addictions (IntNSA). Coordenador do Departamento de Enfermagem Psiquiátrica e Saúde Mental da Associação Brasileira de Enfermagem (ABEn/RJ).

\section{Sarah R. Fonseca Silva}

Mestre em Atenção Psicossocial pelo Instituto de Psiquiatria da Universidade Federal do Rio de Janeiro (UFRJ). Graduada em Psicologia pela Universidade Federal do Vale de São Francisco (Univasf). Coordenadora de Rede de Atenção Psicossocial no município do Rio de Janeiro. Supervisora clínico-institucional. 


\section{RESUMO}

As cenas de uso de drogas no Rio de Janeiro aglutinam uma série de serviços e dispositivos direcionados aos usuários em seus territórios. A Rede de Atenção Psicossocial (RAPS) realiza ações periódicas de cuidado e atenção com os usuários desses espaços, que recebem atendimento e tratamento das equipes profissionais que vão às cenas. O Centro de Atenção Psicossocial Álcool e outras Drogas (CAPS AD) é um dos serviços que realiza ações territoriais, no entanto as dificuldades e precariedades desse trabalho se agravaram diante do contexto pandêmico do coronavírus. Pretendemos, com este texto, apresentar o funcionamento do CAPS AD com a RAPS nas cenas de uso, seus principais desafios, bem como o que foi modificado no manejo do cuidado devido à pandemia da Covid-I9.

Palavras-chave: cena de uso; álcool e outras drogas; CAPS AD; Covid-I9.

\section{ABSTRACT}

More and more, drugs users' environments demand a series of services and public management devices. RAPS (The Psychosocial Health Care Network) usually provides the target public with social actions including localspecific nursing and care, by offering them professional treatment and social assistance and having their needs supplied in their living areas. CAP AD (The Center of Health Care for Alcoholics and Other Addicts) is one of the programs which undertakes local actions in such scenarios. However, as the project has struggled against financial hardships and faced other obstacles along with their performance, the working conditions have grown even more precarious in face of the Coronavirus pandemics. Being so, we intend to present how CAP AD has currently dealt with the aggravating problematics in their working environments, what challenges they have confronted and what has been changed in their care-taking actions owing to the pandemics.

Keywords: drug consumption environments; alcohol and further drugs; CAPS AD; Covid-I9. 


\section{Introdução}

Em 30 de janeiro de 2020, a World Health Organization (202I) declarou a Covid-I9 emergência de saúde pública e, em II de março de 2020, a caracterizou como uma pandemia (WHO, 2020). O Brasil foi o primeiro país da América do Sul a notificar a doença (BURKI, 2020), em I7 de março de 2020, e o Rio de Janeiro entrou oficialmente em estado de emergência por meio de decreto estadual. ${ }^{1}$ Instaurou-se, então, uma grande crise sistêmica.

Nesse contexto, o governo do estado do Rio de Janeiro, em consonância com as recomendações descritas até então, se reorganizou visando mitigar os efeitos da pandemia de Covid-19. Medidas como isolamento social seletivo e ampliado, bem como lockdown foram adotados, entretanto, no decorrer dos meses, tais estratégias se enfraqueceram, apesar do aumento significativo de casos confirmados, óbitos e lotação dos leitos hospitalares (RIO DE JANEIRO, 2020).

A atual pandemia de Covid-I9 produziu não somente efeitos físicos, como também, repercussões psíquicas e sociais avassaladoras. Sintomas como ansiedade, depressão, estresse, automutilação, suicídio e aumento do consumo de álcool e outras drogas são evidenciados (HOLMES et al., 2020), além de dificuldades financeiras, desemprego e incertezas sobre o futuro (REHM et al., 2020).

Por isso, é necessário problematizar as violências que sempre existiram e foram evidenciadas pela pandemia de Covid-I9. Explicita-se as fragilidades de políticas voltadas às pessoas em situação de vulnerabilidade psicossocial, usuários de substâncias psicoativas (SPA) e pessoas em situação de rua. Essa população é atravessada de forma dupla na pandemia, não só pelo uso de SPA, mas também por estarem em situação de rua.

Ressaltar tais questões é colocar em discussão que o cuidado que se desdobra na saúde precisa contemplar esses aspectos, pois a amplitude clínica que se apresenta é também multifacetada. Tanto que Teixeira et al. (2019) salientam que muitas pessoas que estão em situação de rua acabam fazendo uso de álcool e outras drogas como modo de enfrentamento para suportar a vida. Assim, as ações desenvolvidas pelas unidades de saúde de base territorial e comunitária precisam ser intersetoriais e em rede, dada a complexidade dos casos.

Dentro desse cenário, os Centros de Atenção Psicossocial Álcool e Outras Drogas (CAPS $\mathrm{AD}$ ) representam um dos dispositivos que compõem a Rede de Atenção Psicossocial (RAPS). ${ }^{2}$

\footnotetext{
${ }^{1}$ Decreto $\mathrm{n}^{-} 46.973 / 2020$.

${ }^{2}$ A RAPS apresenta-se com os seguintes componentes: Atenção Básica em Saúde (Unidade Básica de Saúde, Consultório na Rua, apoio aos serviços do componente Atenção Residencial de Caráter Transitório e Centros de Convivência); Atenção Psicossocial Especializada (Centros de Atenção Psicossocial em diferentes modalidades, tais como: CAPS I; CAPS II e CAPS AD II; CAPS III e CAPS AD III; e CAPS i); Atenção de Urgência e Emergência (SAMU 192, Sala de Estabilização e UPA 24 horas); Atenção Residencial de Caráter Transitório (Unidade de Acolhimento, Serviço de Atenção em Regime Residencial); Atenção Hospitalar (Enfermaria Especializada em Hospital Geral e serviço hospitalar de referência para atenção às pessoas com sofrimento ou transtorno mental e
} 
Tais serviços prestam assistência integral às pessoas com necessidades decorrentes do uso de álcool e outras drogas, 24 horas, em todos os dias da semana, incluindo finais de semana e feriados (BRASIL, 2OI2).

Para tal, os CAPS AD, por meio das estratégias de Redução de Danos (RD), desenvolvem ações para garantir acesso à saúde, acompanhamento longitudinal, e atenção e acolhimento à crise e a situações de maior risco e vulnerabilidade psicossocial. Visando garantir acompanhamento aos usuários no território dos CAPS AD, a realização do trabalho ocorre não só por meio de visitas domiciliares (VD), como também, nas cenas de uso, ${ }^{3}$ é feito o compartilhamento de experiências e a construção de potencialidades para o cuidado.

Durante a pandemia, entretanto, esses dispositivos necessitaram se reinventar, não mais como serviços substitutivos ${ }^{4}$ aos hospitais psiquiátricos, mas sim como serviços essenciais. Assim, as equipes dos CAPS AD se prepararam para lidar com os desdobramentos pandêmicos dentro da realidade das pessoas assistidas, que apresentavam novas demandas diante do inesperado cenário. Um dos principais desafios era sustentar as mudanças que pediam isolamento, distanciamento social, água e sabão como formas básicas de evitar o contágio. As medidas solicitadas estavam muito distantes do cotidiano dos sujeitos das cenas de uso, cuja existência evocou uma série de medidas particulares. Desse modo, este artigo tem como objetivo relatar a experiência específica do Centro de Atenção Psicossocial Álcool e Outras Drogas Antônio Carlos Mussum (CAPS AD III), na cidade do Rio de Janeiro(RJ), acerca das mudanças que a pandemia trouxe nas ações territoriais nas cenas de uso.

\section{A RAPS e os fluxos da rede}

No Brasil, a Política Nacional de Saúde Mental (PNSM), constituída no processo de Reforma Psiquiátrica Brasileira (RPB), orienta-se a partir da Lei no IO.216/200I (Lei Nacional de Reforma Psiquiátrica), e, atualmente, da Portaria no 3.088/20II, que institui a Rede de

com necessidades decorrentes do uso de crack, álcool e outras drogas); estratégias de desinstitucionalização, como Serviços Residenciais Terapêuticos; e Reabilitação Psicossocial (iniciativas de geração de trabalho e renda, empreendimentos solidários e cooperativas sociais)

${ }^{3}$ Cena aqui entendida, segundo Bastos e Bertoni (2014), "como um conceito socioantropológico referente a um espaço de congregação e interação social presente na literatura em ciências sociais de inspiração tanto anglo-germânica como francesa [...]. As cenas abertas de maior dimensão e maior concentração de usuários passaram a ser denominadas 'cracolândias', termo que se popularizou nos meios de comunicação e na linguagem cotidiana” (p. 18).

${ }^{4}$ Serviços substitutivos se referem às mudanças que a Reforma Psiquiátrica (Lei no 10.226/2001). Conforme explicam Prazeres e Miranda (2005, p. 200-205): "Nos últimos 20 anos, o movimento da Reforma Psiquiátrica no Brasil tem, insistentemente, colocado para a sociedade brasileira a necessidade de se abolir o hospital psiquiátrico como local de tratamento para o portador de sofrimento mental [...] O serviço substitutivo é um dispositivo de atenção em saúde mental que significa, entre outras coisas, a possibilidade de se cuidar das pessoas em sofrimento psíquico sem retirá-las do seu meio, bem como a facilidade maior do mesmo no acesso ao hospital psiquiátrico, principalmente a partir do momento em que este passou a ser a porta de entrada para internações hospitalares. Portanto, o serviço e o hospital fazem parte de um sistema cuja finalidade é a assistência ao portador de sofrimento mental". 
Atenção Psicossocial (RAPS). Em sua implementação, a PNSM incorpora o paradigma de RD, sendo conceituado por Andrade (2OII) como uma estratégia composta de um conjunto de medidas em saúde com o intuito de minimizar as consequências adversas provenientes do uso e abuso de SPA, tendo como fundamento o respeito à liberdade de escolha do indivíduo e o acesso aos serviços de saúde em articulação.

Apesar dos avanços, foi aprovada a Política Nacional sobre Drogas, através do recente Decreto $\mathrm{n}$-9.76I/2019, demarcando o foco na abstinência, destituindo o lugar da RD como política oficial. No entanto, para as pessoas que se mantém na rua, na prática, a principal possibilidade de cuidado permanece localizada na articulação em liberdade de uma rede complexa, tendo em vista um adoecimento multicausal.

Em conferência sobre Determinantes Sociais da Saúde, a World Health Organization (WHO, 2OII) defendeu que os governos se responsabilizem pela criação de redes intersetoriais que possam fazer frente a diversas desigualdades e que se disponibilizem a trabalhar pela saúde de sujeitos que não possam custear o próprio cuidado, desenvolvendo estratégias compatíveis com as características desse público. Portanto, a intersetorialidade se apresenta como resposta concreta em articulação entre rede de saúde e diversos outros setores com o objetivo de incidir com o uso de diferentes tecnologias sobre a qualidade de vida das pessoas (CECÍLIO, 200I).

No setor público, a existência de uma rede de saúde institui um conjunto de serviços que deve atuar de maneira integrada. A categoria de rede opera como uma interpendência dos serviços na concepção de equipamentos, dispositivos e instituições. Age, assim, "como tipo ideal de gestão, arquitetura para o planejamento de uma gestão intersetorial estruturada em rede" (MARTINEZ, 20I6, p. 282). Nessa perspectiva, a RAPS, como foi idealizada, compreende uma ação integradora de casos que devem ser vivenciados intersetorialmente.

A concepção da RAPS considera princípios da universalidade, integralidade e equidade, constitutivos do Sistema Único de Saúde (SUS). Para tal, lança mão de equipe multiprofissional, com abertura de acessos e possibilidades de circuitos, com recursos clínicos para o cuidado integral. A complexidade da rede envolve construir uma relação de ajuste entre autonomia e interdependência, em que cada serviço seja autônomo e responsável por seu funcionamento próprio, tendo parte na tomada de decisões, mas que não deve estar desarticulado com as lógicas que sustentam o corpo institucional.

O conceito de rede é fundamental para entender sentidos e métodos utilizados pelos CAPS, que são serviços de atenção psicossocial estratégica. No caso dos CAPS AD, leva-se em conta diferenças no modo do trabalho. Ainda que a maioria dos serviços opere pela mesma lógica macropolítica instaurada pela RAPS, há a especificidade de ser álcool e outras drogas 
(AD), cujo encaixe com o uso das drogas absorve não somente um circuito institucional, como também pessoas e experiências.

A questão $\mathrm{AD}$ não se trata de um detalhe no tratamento global, e sim de modificação de cenários, contextos e práticas de ação, evidenciando dissonâncias que passam tanto pela percepção de cuidado quanto pelos manejos e formas de tratamento. O CAPS AD, por sua estratégia de cuidado, norteia as práticas de saúde, a partir das relações estabelecidas no consumo de drogas. É um serviço de saúde de caráter híbrido, que deve lançar mão de estratégias de atenção direcionadas para o cuidado, podendo provocar interpretações, por vezes dissonantes, entre profissionais, como psiquiatras, psicólogos, redutores de danos, enfermeiros etc.

O enfoque recai no entendimento da RAPS como um arranjo institucional que compreende equipamentos e serviços. Essa compreensão se dá por meio dos itinerários terapêuticos, tanto dos usuários, quanto dos profissionais, e as redes que devem se mobilizar para funcionar. Se por um lado há profissionais acionando redes para tornar seus trabalhos efetivos, usuários circulam e são absorvidos por essa malha para tornar suas vidas possíveis.

Por sua vez, os CAPS AD se estabelecem entre circuitos, estando presentes dentro de um tecido urbano que reúne política sanitária, de drogas, de segurança e de assistência, se solidificando na lógica construída pelos usuários, em que o Estado, por meio de instituições, valida percursos, escolhas e ações. A possibilidade articuladora do CAPS AD em toda essa ordem nos indica que "processos políticos transinstitucionais de rede não são como constructo simbólico para pensar a estrutura das relações sociais, mas como instrumento de compreensão das relações entre indivíduos" (BARNES ,I988, p. 86).

A ideia de relações dialoga com a pesquisa sobre os Consultórios na Rua, de Mariana Martinez (2016), em que atuar em rede conecta "materialidade, canal de escoamento, troca, fluxo. Mais que aliança, passam por gente, registros, informações, afetos, recursos materiais" (p. 285). A gestão do cuidado materializada na ideia de rede se dá devido aos recursos partilhados, e ocorre junto com uma "gestão populacional dos marginais em que a lógica de punição, repressão e controle convive com preocupações de assistência, saúde que acionam equipamentos, discursos, saberes e poderes" (MALLART; RUI, 2015, p. IO).

Coexistem, portanto, dispositivos de gestão da ordem e do cuidado com trânsitos incessantes. São os trânsitos dos usuários por sistema de justiça, de assistência, de saúde e de religião que dão a potencialidade de conectar territórios, antes não pensados como atuando mutuamente, a despeito de suas diferentes premissas e ações. O fato é que os usuários promovem ressonâncias entre os serviços para cada um de seus cotidianos e os gestam conectando pontas da rede de saúde com dispositivos intersetoriais. 


\section{Ações territoriais de saúde mental nas cenas de uso}

O trabalho da atenção psicossocial tem uma atuação de base territorial e comunitária, assim a articulação profissional e em rede pressupõe o território em que estão, o que se denomina como missão territorial. Essa nomenclatura se refere a um tratamento complexo com gestão participativa, que envolve a ação de vários profissionais de saúde, usuários e a comunidade a qual se inscrevem.

A Secretaria Municipal de Saúde (SMS) do Rio de Janeiro divide a cidade em Áreas Programáticas (AP). Essa divisão geográfica, como apresentada na Figura I, permite uma organização dos dispositivos que compõem a RAPS de modo a viabilizar acesso, acompanhamento e acolhimento de base territorial e comunitária. Assim, o território, com suas dinâmicas e vivacidades, necessita ser compreendido para além da organização espacial. Inclui-se aqui uma dimensão subjetiva que faz com que usuários estabeleçam relações geradoras de vida ou de constante vulnerabilização.

\section{Figura 1. Áreas Programáticas (AP) do Rio de Janeiro.}

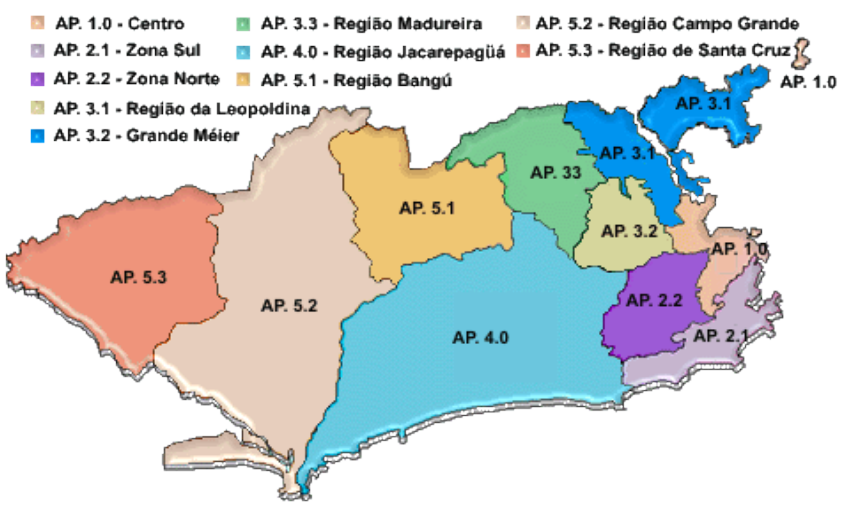

Fonte: Prefeitura da Cidade do Rio de Janeiro (2017). Disponível em:<http://www.rio.rj.gov.br/documents/73801/2e652a52-c1a3-4de3-a2bd-e80eefc0280a>-

Essa dimensão é essencial para os CAPS, tendo em vista a necessidade de trabalhar o território como potência no cuidado aos usuários. Os CAPS assumem, então, uma função que não corresponde a de punição, como majoritariamente respondem as políticas repressivas às questões de álcool e outras drogas; e nem de culpa, a exemplo de algumas organizações religiosas; mas sim de mediação das relações que são possíveis para sustentar o cuidado no próprio território. 
De acordo com FAGUNDES et al. (2016), os CAPS AD não estão localizados necessariamente nas regiões onde há cena de uso de drogas, contudo esses serviços se deslocam para essas cenas, realizando junto à rede, em média, 8 mil atendimentos por mês. Além dos CAPS AD há outros serviços que promovem ações nesses espaços - como o Consultório na Rua ( $\mathrm{CnR}$ ) - e equipamentos da assistência social, por exemplo. Além dos dispositivos RAPS e/ou vinculados à Secretaria Municipal de Assistência Social e Direitos Humanos (SMASDH), tais territórios recebem um fluxo de outras iniciativas, como ONGs, igrejas e representantes de Comunidades Terapêuticas (CTs).

No que se refere aos usuários, há uma grande dificuldade de chegada e adesão aos serviços, o que expressa as peculiaridades desse tipo de clientela e que impõe a necessidade de flexibilização das práticas instituídas. Grande parte dos usuários vinculados aos CAPS AD é atendida na própria cena. Como os CAPS atuam dentro dos critérios da autonomia do usuário, o desafio posto é de dar prosseguimento a um cuidado que produza estratégias de aumentar a adesão.

Importante salientar também a maneira como alguns profissionais acolhem usuários da cena de uso dentro do CAPS AD. Há entre a equipe multiprofissional uma atenção a dois pontos das atividades territoriais: um referente ao que chamam de esvaziamento da equipe; o outro, de que usuários da cena não têm o mesmo entendimento que os outros usuários do serviço, e acabam tumultuando o cotidiano de trabalho. No entanto, esse posicionamento é rebatido por outros profissionais e pela própria gestão ao reiterarem as missões territoriais da atenção psicossocial e ao afirmarem que o CAPS, quando sai da unidade de saúde mental, continua sendo CAPS em ação.

O debate entre os profissionais acerca das ações territoriais em cenas de uso no CAPS AD é muito intenso, vai desde a frequência das visitas; a dificuldade de trazer usuários para o serviço; as particularidades da presença de crianças, adolescentes e mulheres grávidas; até como envolve criação e manutenção de vínculos nesse processo. Outra questão se refere à relação com o judiciário, ao passo que a saúde pode pautar a justiça, visto que um médico pode dizer que não é possível alguma medida para certo indivíduo.

As cenas de uso trazem um novo paradigma de cuidado que estabelece uma esfera sobre a possibilidade de cuidar do usuário estando no território dele. Agenciar a rede do usuário em seu espaço é uma das oportunidades de acessar um repertório de cuidado em saúde, onde se apresenta, também, a rede do sujeito. Conhece-se o bar, o vizinho, a associação de moradores e a clínica da família, por exemplo. 
A cidade do Rio de Janeiro não tem um número fixo de cenas de uso, pois são muitas delas itinerantes e dependem, em grande medida, da facção do tráfico ou da milícia que está no comando. Estão submetidas, também, às políticas públicas que podem ser acionadas no espaço. Há ainda a discussão sobre recolhimento que reascende o debate sobre higienização social.

\section{As cenas de uso de drogas no Rio de Janeiro}

As cenas de uso são um epicentro em que se visualiza quais redes sustentam os serviços. Uma das características é que unem, num mesmo lugar, quem trabalha e quem não trabalha com a RD, fazendo confrontar métodos de abordagens, já que diariamente chegam às cenas: o Estado, em forma de saúde, de assistência social, de justiça e de limpeza urbana; como também a associação dos moradores, a igreja, as ONGs e, é claro, o crime organizado local, com sua determinada facção ou milícia. Políticas públicas e iniciativas da sociedade civil convivem corpo a corpo num lócus de associações de rede.

O cenário une uma equipe multidisciplinar com variadas formações e usuários com trajetórias de vida e visões familiares (ainda atuantes e fortes em tomadas de decisão individuais) - visões que contam com a presença da referência de preconceito com a substância e seu usuário. Soma-se a toda essa complexidade de atores aqueles que dominam e definem o que é e como vai ser o território, que determinam as regras de mercado e varejo na economia real e nas economias morais.

O Rio de Janeiro apresenta particularidades que não devem ser ignoradas, pois interferem na vida social da cidade como um todo e na especificidade cotidiana de existência das cenas de uso. A cada novo rumo tomado pelas facções ou pelo Estado - se acontece um confronto maior com a polícia, se a violência se aprofunda na troca de tiros -, a dinâmica das cenas é afetada e, consequentemente, as ações territoriais dos serviços de saúde também o são. Não são raras as vezes que os CAPS AD devem suspender à ida ao território devido à violência, sendo esse o fator de mais dificuldade para a continuidade dos projetos.

A relação do Rio de Janeiro com o comércio e o uso do crack foi conflituosa desde seu início, diferentemente de cidades como São Paulo, que no ano 2000 tinha uma importância no mercado ${ }^{5}$ e no varejo do crack, e na formação da cracolândia (RUI, 20I4). Já no Rio, a droga

\footnotetext{
5 "Com efeito, foi a criação de uma cena de consumo mais fixada que fez emergir ações assistenciais e sanitárias do Estado. Além disso, entre janeiro e julho de 2015, um novo momento se demarca a partir da efetiva consolidação do interesse da ONG Redes da Maré pelo local. Em parceria com o Centro de Estudos de Segurança e Cidadania (CESeC) e com o Núcleo Interdisciplinar de Ação e Cidadania (NIAC/UFRJ), com apoio financeiro da Open Society Foundation (OSF), uma equipe da ONG passa a atuar diretamente no local, inicialmente fazendo um levantamento da população fixada e, com o transcorrer das atividades, oferecendo oficinas de fotografia e um cineclube aos usuários da droga. Produzindo conhecimento mais qualificado sobre o local, o levantamento realizado enumera que, no ano de 2015, havia cerca de setenta moradores fixos, distribuídos em dezesseis barracos. Observa também que não há possibilidade de expansão dessa cena de consumo e que o 'direito à moradia' é regulado, mas que há certa rotatividade de pessoas chegando e saindo" (RUI, 2019, p. 585).
} 
só despontou como uma questão entre 2012 e 2013, quando usuários de crack foram vistos na Avenida Brasil, em um momento simbólico para o país e para a cidade devido aos megaeventos internacionais - Copa das Confederações (2013), Copa do Mundo (2014) e Olimpíadas (2016) - e à chegada das Unidades de Polícia Pacificadora (UPP). À tamanha visibilidade que tomou a presença desses usuários na Avenida Brasil e em favelas como Jacarezinho e Manguinhos também se conecta a ocupação militar do Complexo do Alemão (FRÚGOLI JR.; CAVALCANTI, 2OI3).

Além da conjuntura política, dinâmicas e mudanças dentro das facções facilitaram a entrada do crack no Rio de Janeiro, mesmo que considerada tardia para alguns. Megaeventos, UPP e enfraquecimento do Comando Vermelho (CV)(MISSE, 2003, 20II), a facção dominante até então, foram as principais portas de entrada para o crack na cidade, o qual por muito tempo foi vetado pelo CV por movimentar o lucro de maneira pouco atrativa; por ser disseminado pela mídia como uma substância muito destrutiva, atraindo holofotes para usuários denominados "zumbis" e com "forte a pelo moral, além de comprometer relações de vizinhança com pequenos furtos" (RUI, 2019, p. 584).

No entanto, ainda que tais argumentos fossem fortes, eles conviviam com a expansão visível e a demanda do consumo. Em resposta a essa tomada de território pelos usuários de crack, houve uma grande aposta higienista com políticas municipais de recolhimento compulsório onde reiniciaram seus "esforços territorializantes" (FRÚGOLI JR.; CAVALCANTI, 20I3), e o Complexo da Maré foi um dos principais destinos, na extensão entre Parque União, Rubens Vaz, Nova Holanda e Parque Maré. Segundo pesquisa realizada na Maré, Taniele Rui (2014) narra o seguinte:

O interessante dessa história, que presenciei, é que foi acordado entre a associação dos moradores, o CV e usuários que estes deixassem a Avenida Brasil e passassem a ocupar uma esquina da favela, a fim de fazer diminuir as incursões policiais. O lugar para onde foram levados era uma área liminar, estruturada de antigos depósitos industriais e apartada das áreas residenciais e das ruas comerciais. Inicialmente, a cena de uso constituía-se de não mais que quinze barracos de lona preta que abrigavam os consumidores, muitos dos quais não eram da Maré. Eles ficavam por ali apenas durante o dia, mas voltavam para a avenida durante a noite, já que a esquina ficava exatamente na divisa estabelecida internamente como demarcação entre o CV e o Terceiro Comando (TC). (p. 583) 
Em territórios como esses, os CAPS AD investem na construção de relações com a comunidade que demarquem a importância da perspectiva da $\mathrm{RD}$, articulada com diversos dispositivos, formais ou informais. A ação nas cenas de uso atinge um território inteiro, mostrando que a ação semanal tem desdobramentos em muitos setores. Portanto, ocupar o território ofertando cuidado ressignifica a relação com a comunidade.

\section{Cenas de uso no contexto da pandemia}

A chegada da crise sanitária causada pela Covid-ı com consequente intensificação da fome, redução da circulação de transeuntes e posterior enfraquecimento do comércio local, desde o primeiro lockdown, mostrou, ainda no início da pandemia, que a população em situação de rua, já muito mais vulnerabilizada pela pobreza do que a média, corria mais riscos de infecção e agravos decorrentes dela.

Dentro do serviço, principalmente nos três primeiros meses, notou-se uma redução da presença do público em geral em virtude das restrições de circulação pela cidade e da orientação para evitar aglomerações no CAPS AD. Foram pactuados modos de espaçar a frequência de idas à unidade, bem como a inauguração de atendimentos remotos, entre outras estratégias que visassem garantir o cuidado ao mesmo tempo que reduzia os riscos de contágio.

Nas ruas, rapidamente observou-se com preocupação a fragilização das redes de suporte, que habitualmente viabilizavam o suprimento de comida e outros donativos. Mesmo o retorno à casa dos familiares, que era comum entre esse público, passou a ser impedido para que o trânsito dessas pessoas não as transformasse em vetores de transmissão. Por esse motivo, muitos daqueles que frequentavam a cena de uso, mas voltavam para casa para dormir, passaram a permanecer na rua. Esses aspectos fizeram com que os indivíduos acompanhados nesses cenários passassem a comparecer mais ao serviço em busca de comida ou mesmo de acolhimento.

Nesse contexto, os profissionais do CAPS AD novamente trouxeram a questão da ida até a cena, já que o risco de infecção na insalubridade desse espaço era aumentado, mas, em contrapartida, entenderam que, sendo a principal porta de acesso à rede de saúde para aquela população, não seria possível ser interrompida. Assim, alinhados ao princípio da universalidade e da equidade (BRASIL, I990), foram convocados a manter o acompanhamento desses usuários empregando estratégias de $\mathrm{RD}$ adaptadas à conjuntura.

Rapidamente, o uso da máscara precisou ser imposto. Inseriu-se, então, como nova estratégia de $\mathrm{RD}$, a disponibilização da máscara cirúrgica como componente nos kits de RD. Sua distribuição visava tanto proteger os profissionais e reduzir a transmissão de vírus, sobretudo da Covid-19, quanto ajudar os usuários a transitar pela cidade, acessando seus 
pontos de apoio que se tornaram mais escassos, na medida em que a pandemia e concomitantemente a crise econômica foram avançando.

As equipes do CAPS AD se mantêm visitando as cenas e, nessas ocasiões, é comum que sejam encontradas pessoas com sintomas da Covid-I9, algumas relatando já terem buscado suas Unidades Básicas de referência ou mesmo hospitais, e muitas se queixando de não terem tido acompanhamento intensificado diante dos quadros que se apresentavam associados à vulnerabilidade social. Se em outros momentos, o fato de serem pessoas em situação de rua não alterava a conduta das equipes de saúde, na pandemia isso se inviabilizou ainda mais, uma vez que, diante da saturação do sistema de saúde, a pobreza, a fome ou a ausência de possibilidades de se isolar raramente influenciavam as avaliações de risco, que eram pautadas apenas na sintomatologia.

Associando condições de vida e saúde, a World Health Organization (WHO, 2OII), na Conferência sobre Determinantes Sociais da Saúde, destacou que:

\footnotetext{
As iniquidades em saúde são um problema em todos os países e refletem não só as disparidades de renda e riqueza, como também diferenças nas oportunidades dadas aos indivíduos com base em fatores como etnia, raça, classe, gênero, nível educacional, deficiências, orientação sexual e localização geográfica. Essas diferenças produzem graves consequências e representam uma forma de impacto que se chama "determinantes sociais da saúde".(p. I)
}

Esses determinantes representam injustiças firmadas em um modo de desigualdade que coloca alguns grupos populacionais em desvantagem quanto à possibilidade de se manterem sadios, e devem ser levados em consideração na produção do cuidado.

No entanto, se uma das pessoas vinculadas às cenas buscavam um hospital apresentando tosse, febre e esforço respiratório, a ela era recomendado que retornasse para casa e repousasse. Ainda que se argumentasse a ausência de casa, a conduta se mantinha, desta vez justificada também pela superlotação das unidades hospitalares. Porém, episódios como esses historicamente se reproduziram antes mesmo da pandemia, sustentados na contramão da noção de determinação social da saúde. Na prática, é mantido um fazer construído para pessoas que usufruem do mínimo necessário que não dialoga com realidades como as da rua.

Nesse cenário, grande parte dos profissionais de saúde que atuam nos hospitais não entendem a questão da moradia como algo que lhes diga respeito; por outro lado, os abrigos passam a atuar de maneira ainda mais restritiva frente à pandemia, tendo em vista cuidados com biossegurança e o imperativo de evitar aglomerações, sobretudo se existe a possibilidade 
de algum dos sujeitos estar infectado. As idas das equipes do CAPS AD até as cenas acabam se desdobrando na articulação desses casos com a rede de saúde e de assistência social.

Se no agravamento dos sintomas o acesso era limitado, em outras situações normalmente as pessoas com sintomas leves de síndrome gripal, ao buscarem unidades de saúde, tinham também como orientação cuidados domiciliares, por isso não permaneciam e, em seguida, os quadros se agravavam, possivelmente condicionados pela insalubridade à qual estavam submetidas. Assim, os profissionais foram convocados a viabilizar cuidado condizente com a realidade de cada sujeito, sem apartá-lo do quadro clínico, ora acionando hospitais e unidades de atenção primária, ora oferecendo a permanência nos leitos de atenção à crise.

\section{Considerações finais}

Conforme apresentado aqui, as ações territoriais nas cenas de uso são essenciais dentro do modelo de cuidado e atenção do CAPS AD. No entanto, há dificuldades nas práticas de tratamento nas cenas que se intensificaram com a chegada da pandemia. As equipes dos CAPS AD precisaram se preparar para lidar com as mudanças causadas pela pandemia de Covid-I9, que modificou a realidade das pessoas assistidas, evidenciando novas demandas. Como foi explicitado, um dos principais desafios foi sustentar as regras de isolamento, distanciamento social, e água e sabão como formas básicas de evitar o contágio, pois estavam muito distantes do cotidiano dos usuários das cenas de uso.

Assim, os CAPS AD passaram a responder cada vez mais à crise relativa à fragilização de uma rede - tanto formal, quanto informal - que viabilizava condições mínimas para promoção de saúde nos contextos em que estão inseridos os usuários do serviço. Ainda que a solidariedade seja percebida com frequência nas comunidades nas quais estão localizadas as cenas visitadas, o empobrecimento da população em geral, e muito mais das pessoas em situação de rua, fez com que fosse necessário recorrer com maior frequência a recursos de alta complexidade da rede nos quais estão localizados os leitos de acolhimento à crise e os hospitalares (BRASIL, 2006). Desse modo, o tão falado colapso causado pela pandemia de Covid-I9 e pelas respostas governamentais a ela, foi sentido não apenas pelas equipes das unidades hospitalares envolvidas diretamente com a doença, mas também por equipes de saúde mental.

Diante desse quadro, o que antes já se colocava como desafio, como o diálogo e o trabalho integrado da rede voltado para os casos mais dramáticos das cenas de uso, se radicaliza no cenário pandêmico de isolamento, num contexto de sintomas pouco específico e com pouco 
conhecimento sobre as consequências da doença. A chegada da Covid-I9 intensificou tensões entre os fluxos da rede e fez com que as equipes recalculassem rotas de cuidado para que o tratamento destinado a essa população conseguisse ser efetivo.

\section{Lista de siglas}

AP - Área Programática

CAPS - Centro de Atenção Psicossocial

CAPS AD - Centro de Atenção Psicossocial Álcool e outras Drogas

CESeC - Centro de Estudos de Segurança e Cidadania

CnR - Consultório na Rua

CT - Comunidade Terapêutica

IPEA - Instituto de Pesquisa Econômica Aplicada

NIAC - Núcleo Interdisciplinar de Ações para a Cidadania

OMS - Organização Mundial de Saúde

ONG - Organização Não Governamental

OSF - Open Society Foundation

RD - Redução de Danos

SMS - Secretaria Municipal de Saúde

SMASDH - Secretaria Municipal de Assistência Social e Direitos Humanos

SPA - Substâncias Psicoativas

SUS - Sistema Único de Saúde

UPP - Unidade de Polícia Pacificadora

VD - Visita Domiciliar

WHO - World Health Organization

\section{Referências}

ANDRADE, T. Reflexões sobre políticas de drogas no Brasil. Ciência e Saúde Coletiva, v. I6, p. 4665$4674,2011$.

BARNES, J. A. Redes sociais e processo político. In: FELDMAN BIANCO, B. (Org.). Antropologia das sociedades contemporâneas. São Paulo: Global, 1988. 
BASTOS, F. I. ; BERTONI, N. (Orgs.). Pesquisa Nacional sobre o uso de crack: quem são os usuários de crack e/ou similares do Brasil? Quantos são nas capitais brasileiras? Rio de Janeiro: ICICT/Fiocruz, 2014.

BRASIL. Decreto n-9.76I, de II de abril de 20I9. Aprova a Política Nacional sobre Drogas. Diário Oficial [da] República Federativa do Brasil, Brasília, DF, I2 abr. 2019.

BRASIL. Lei n- IO.216, de 6 de abril de 200I. Dispõe sobre a proteção e os direitos das pessoas portadoras de transtornos mentais e redireciona o modelo assistencial em saúde mental. Diário Oficial [da] República Federativa do Brasil, Brasília, DF, 7 abr. $200 \mathrm{I}$.

BRASIL. Lei no 8.080, de I9 de setembro de 1990. Lei Orgânica da Saúde. Dispõe sobre as condições para a promoção, proteção e recuperação da saúde, a organização e o funcionamento dos serviços correspondentes e dá outras providências. Diário Oficial [da] República Federativa do Brasil, Brasília, DF, I9 set. I990.

BRASIL. Ministério da Saúde. Pactos pela vida, em defesa do SUS e de gestão - diretrizes operacionais. Brasília, DF: Departamento de Apoio à Descentralização/Secretaria Executiva, 2006. (Série Pactos Pela Saúde 2006, I).

BRASIL. Ministério da Saúde. Portaria nํㅛ 130, de 26 de janeiro de 20I2. Redefine o Centro de Atenção Psicossocial de Álcool e outras Drogas 24 h(CAPS AD III) e os respectivos incentivos financeiros. Diário Oficial [da] República Federativa do Brasil, Brasília, DF, 26 jan. 2012.

BRASIL. Ministério da Saúde. Portaria no 3.088, de 23 de dezembro de 20II. Institui a Rede de Atenção Psicossocial para pessoas com sofrimento ou transtorno mental e com necessidades decorrentes do uso de crack, álcool e outras drogas, no âmbito do SUS. Diário Oficial [da] República Federativa do Brasil, Brasília, DF, 24 dez. 201 I.

BURKI, T. Covid-I9 in Latin America. Lancet Infect Dis., v. 20, n. 5, p. 547-548, or maio 2020. Disponível em: $<$ https://www.thelancet.com/journals/laninf/article/PIISI4733099(20)30303-0/fulltext>. Acesso em: OI maio 202I.

CECÍLIO, L.C.O. As necessidades de saúde como conceito estruturante na luta pela integralidade e equidade na atenção em saúde. In: PINHEIRO, R.; MATTOS, R. A. (Org.). Os sentidos da integralidade. Rio de Janeiro: Abrasco, 200I. p. II3-I26.

FAGUNDES JR., H. M. et al. Reforma psiquiátrica no Rio de Janeiro: situação atual e perspectivas futuras. Ciência e Saúde Coletiva. Rio de Janeiro, v. 2I, n. 5, p. I449-I460, 2016. Disponível em: $<\mathrm{http}: / /$ www.scielo.br/pdf/csc/v2In5/I4I3-8I23-csc-2I-05-I449.pdf >. Acesso em: 08 maio $2 \mathrm{O} 2 \mathrm{I}$.

FRÚGOLI JR., H.; CAVALCANTI, M. Territorialidades da(s) cracolândia(s) em São Paulo e Rio de Janeiro. Anuário Antropológico/2OI2, v. 38, n. 2, 2013. 
HOLMES, E. A. et al. Multidisciplinary research priorities for the Covid-I9 pandemic: a call for action for mental health Science. Lancet Psychiatry, v. 7, n. 6, p. 547-560, jun. 2020. Disponível em: $<$ https://www.thelancet.com/journals/lanpsy/article/PIIS22I5-0366(20)30I68-I/fulltext $>$. Acesso em: 08 maio 202I.

MALLART, F.; RUI, T. C. Por uma antropologia das transversalidades urbanas: entre o mundão e os dispositivos de controle. In: ENCONTRO ANUAL DA ANPOCS. GT 34 - Sobre Periferias: Novos Conflitos no Espaço Público, 39., 2015, Caxambu. Anais... Caxambu, 2015.

MARTINEZ, M. O Consultório na Rua e as novas formas de intervenção em cenários de uso do crack: o caso de São Bernardo do Campo. In: RUI, T. et al.(Orgs.). Novasfaces da vida nas ruas. São Carlos: EdUFSCar, 2016.

MISSE, M. O movimento: a constituição e reprodução das redes do mercado informal ilegal de drogas a varejo no Rio de Janeiro e seus efeitos de violência. In: BAPTISTA, M. et al.(Orgs.). Drogas e pós-modernidade. Vol. 2. Rio de Janeiro: EdUERJ, 2003.

MISSE, M. Os rearranjos de poder no Rio de Janeiro. Le Monde Diplomatique Brasil, Edição 48, or jul. 20II. Disponível em: $<$ https://diplomatique.org.br/os-rearranjos-de-poder-no-rio-dejaneiro/>. Acesso em: 02 maio 2O2I.

PRAZERES, P. S.; MIRANDA, P. S. C. Serviço substitutivo e hospital psiquiátrico: convivência e luta. Psicol. cienc. prof., Brasília, v. 25, n. 2, p. 198-2II, jun. 2005. Disponível em: $<$ http://pepsic.bvsalud.org/scielo.php? script=sci_arttext\&pid=SI4I4$98932005000200004 \& \operatorname{lng}=$ pt\&nrm=iso $>$. Acesso em: 02 maio 2O2I.

REHM, J. et al. Alcohol use in times of the Covid-I9: implications for monitoring and policy. Drug Alcohol Rev., v. 39, n. 4, p. 30I-304, maio 2020. Disponível em: $<$ https://onlinelibrary.wiley.com/doi/full/IO.IIII/dar.I3074>. Acesso em: 02 maio 202I.

RIO DE JANEIRO (Estado). Centro de Pesquisa do Ministério Público. Panorama da pandemia de Covid-I9 no estado do Rio de Janeiro e a necessidade do isolamento social. Rio de Janeiro, maio 2020.

RUI, T. Nas tramas do crack: etnografia da abjeção. São Paulo: Terceiro Nome, 2014.

RUI, T. Por entre territórios visíveis e territórios invisibilizados: mercados ilícitos e cracolândias de São Paulo e Rio de Janeiro. Novos Estudos CEBRAP, São Paulo, v. 38, n. 3, p. 573-588, dez. 2019.

TEIXEIRA, M. B. et al. Os invisibilizados da cidade: o estigma da população em situação de rua no Rio de Janeiro. Revista do Centro Brasileiro de Estudos de Saúde, Rio de Janeiro, v. 43, número especial 7, dez. 2019.

WHO - WORLD HEALTH ORGANIZATION. Timeline: WHO's Covid-I9 response. Geneva, 2O2I. Disponível em: $<$ https://www.who.int/emergencies/diseases/novel-coronavirus2019/interactive-timeline\#>. Acesso em: 29 jun. 202I. 
WHO - WORLD HEALTH ORGANIZATION. Director-Gernal's opening remarks at the media briefing on Covid-I9. Geneva, 2020. Disponível em: $<$ https://www.who.int/director-

general/speeches/detail/who-director-general-s-opening-remarks-at-the-media-briefingon-covid-I9---II-march-202O>. Acesso em: 29 jun. 202I. 